Cochrane Database of Systematic Reviews

\title{
Cytoreductive surgery alone or combined with hyperthermic intraperitoneal chemotherapy (HIPEC) for pseudomyxoma peritonei (Protocol)
}

Pasquali S, Sommariva A, Mahteme H, Suo T, Ma H, Tropea S, Steenberg JL, Mocellin S

Pasquali S, Sommariva A, Mahteme H, Suo T, Ma H, Tropea S, Steenberg JL, Mocellin S.

Cytoreductive surgery alone or combined with hyperthermic intraperitoneal chemotherapy (HIPEC) for pseudomyxoma peritonei. Cochrane Database of Systematic Reviews 2018, Issue 10. Art. No.: CD005659.

DOI: 10.1002/14651858.CD005659.pub3.

www.cochranelibrary.com

Cytoreductive surgery alone or combined with hyperthermic intraperitoneal chemotherapy (HIPEC) for pseudomyxoma peritonei 


\section{TABLE OF CONTENTS}

HEADER . . . . . . . . . . . . . . . . . . . . . . . . . . . . . . . . . . . . . . . . 1

ABSTRACT . . . . . . . . . . . . . . . . . . . . . . . . . . . . . . . . . . . . . . . 1

BACKGROUND . . . . . . . . . . . . . . . . . . . . . . . . . . . . . . . . . . . . 1

OBJECTIVES . . . . . . . . . . . . . . . . . . . . . . . . . . . . . . . . . . . . . .

METHODS . . . . . . . . . . . . . . . . . . . . . . . . . . . . . . . . . . . . . . .

ACKNOWLEDGEMENTS . . . . . . . . . . . . . . . . . . . . . . . . . . . . . . . . . . . . . . .

REFERENCES . . . . . . . . . . . . . . . . . . . . . . . . . . . . . . . . . . . . . . 4

APPENDICES . . . . . . . . . . . . . . . . . . . . . . . . . . . . . . . . . . . . . 10

WHAT'S NEW . . . . . . . . . . . . . . . . . . . . . . . . . . . . . . . . . . . . . . 10

CONTRIBUTIONS OF AUTHORS . . . . . . . . . . . . . . . . . . . . . . . . . . . . . . . . . . 11

DECLARATIONS OF INTEREST . . . . . . . . . . . . . . . . . . . . . . . . . . . . . . . 11

Cytoreductive surgery alone or combined with hyperthermic intraperitoneal chemotherapy (HIPEC) for pseudomyxoma peritonei 
[Intervention Protocol]

\title{
Cytoreductive surgery alone or combined with hyperthermic intraperitoneal chemotherapy (HIPEC) for pseudomyxoma peritonei
}

\author{
Sandro Pasquali ${ }^{1}$, Antonio Sommariva ${ }^{2}$, Haile Mahteme ${ }^{3}$, Tao Suo ${ }^{4}$, Huaixing Ma ${ }^{5}$, Saveria Tropea ${ }^{2}$, Josephine L Steenberg ${ }^{6}$, Simone \\ Mocellin ${ }^{2,7}$

\begin{abstract}
${ }^{1}$ Sarcoma Service, Fondazione IRCCS Istituto Nazionale dei Tumori, Milano, Italy. ${ }^{2}$ Surgical Oncology Unit, Istituto Oncologico Veneto, IOV-IRCCS, Padova, Italy. ${ }^{3}$ Uppsala Cancer Clinic, Uppsala Cancer Clinic, Uppsala, Sweden. ${ }^{4}$ Department of General Surgery, Institute of General Surgery, Zhongshan Hospital, Fudan University, Shanghai, China. ${ }^{5}$ Department of Medical Oncology, Anhui Provincial Cancer Hospital, the First Affiliated Hospital of University of Science and Technology of China (The Western Area), Hefei, China. ${ }^{6}$ Cochrane Colorectal Cancer Group, Bispebjerg Hospital, Region H, Copenhagen NV, Denmark. ${ }^{7}$ Department of Surgery Oncology and Gastroenterology, University of Padova, Padova, Italy
\end{abstract}

Contact address: Simone Mocellin, Surgical Oncology Unit, Istituto Oncologico Veneto, IOV-IRCCS, Via Gattamelata 64, Padova, Veneto, 35128, Italy. simone.mocellin@unipd.it, mocellins@hotmail.com.

Editorial group: Cochrane Colorectal Cancer Group.

Publication status and date: Amended to reflect a change in scope (see 'What's new'), published in Issue 10, 2018.

Citation: Pasquali S, Sommariva A, Mahteme H, Suo T, Ma H, Tropea S, Steenberg JL, Mocellin S. Cytoreductive surgery alone or combined with hyperthermic intraperitoneal chemotherapy (HIPEC) for pseudomyxoma peritonei. Cochrane Database of Systematic Reviews 2018, Issue 10. Art. No.: CD005659. DOI: 10.1002/14651858.CD005659.pub3.

Copyright (C) 2018 The Cochrane Collaboration. Published by John Wiley \& Sons, Ltd.

\section{A B S T R A C T}

This is a protocol for a Cochrane Review (Intervention). The objectives are as follows:

The aim of this work is to evaluate the efficacy of cytoreductive surgery alone versus cytoreductive surgery in combination with hyperthermic intraperitoneal chemotherapy (HIPEC), on patient benefits, complications and short-term outcomes in patients with pseudomyxoma peritonei (PMP).

\section{B A C K G R O U N D}

\section{Description of the condition}

Pseudomyxoma peritonei (PMP) is a rare tumour affecting approximately one person in two millions each year (DeSantis 2017; Smeenk 2008; Sherer 2001; Hinson 1998). PMP is characterised by mucinous ascites (i.e., jelly-like liquid within the abdominal cavity) and peritoneal tumours (i.e., cancers arising from the peritoneum, a thin membrane that lines the abdominal cavity) most commonly originating from a ruptured mucinous tumour of the appendix (Mukherjee 2004; Panarelli 2011; Ronnett 1997; Smeenk 2008a; Young 2004), but occasionally could originate from other mucinous tumours in the abdomen (e.g. in the colon, rectum, stomach, pancreas). Typically these tumours grow slowly within the abdominal cavity without spreading through the blood stream to distant sites (Moran 2003; Ronnett 2001; Smith 1992; Sugarbaker 1996a).

PMP rarely originates from a primary ovarian cancer, and in this

Cytoreductive surgery alone or combined with hyperthermic intraperitoneal chemotherapy (HIPEC) for pseudomyxoma peritonei 
case it presents as a mature teratoma (i.e., an usually benign tumor arising from ovarian germ cells) with aspects of a mucinous neoplasm (i.e., a tumor that produces a jelly-like material) (McKenney 2008). Primary ovarian mucinous tumours are similar to metastasis from appendix mucinous neoplasms (i.e., tumors arising from the colonic appendix), though they have distinct pathological features (Stewart 2014). Other mucinous tumours - such as those from the colon, urachus, and pancreas - may share a PMP presentation (Carr 2012), but they usually progress much more quickly with consequent worse prognosis. Over time, accumulation of mucin in the peritoneal cavity leads to massive symptomatic abdominal distension and ultimately mechanical gastrointestinal obstruction, the main cause of death in these patients.

The definition of PMP has been debated for many years (De Vita 2008). Recently, experts of the Peritoneal Surface Oncology Group International (PSOGI) agreed to consider PMP a clinical syndrome characterised by accumulation of mucinous material in the abdominal cavity, typically sustained by low-grade epithelial tumours growing in a sparse pattern (Carr 2016). Mucinous appendiceal neoplasia are classified as: 1) "mucinous adenocarcinoma" that are lesions with infiltrative growth pattern, 2) "low-grade appendiceal mucinous neoplasm" that are the common low-grade tumours with no infiltrative growth pattern of the appendix, and 3) "high-grade appendiceal mucinous neoplasm" that are lesions showing high-grade cytologic atypia (i.e., abnormal microscopic appearance of the cells composing the tumor) and no infiltrative invasion but with high-grade cytologic atypia. On the other hand, PMP is defined as a syndrome characterised by the "redistribution phenomenon" (Sugarbaker 1994), that is the redistribution through the peritoneal cavity of the mucus and the cells it contains which follow the normal flow of peritoneal fluid to sites of fluid absorption through lymphatic lacunae and lymphoid aggregates. As a consequence, tumour deposits generally are not detected at mobile small bowel loops and accumulates in the pelvis, paracolic gutters, omentum, and liver capsule. However, this new classification also recognises that PMP can harbour different degrees of malignancy, with low-grade through to high-grade histologic features presenting clinically as PMP (Carr 2016). Overall, three main categories of PMP have been identified which are characterised by low-grade, high-grade, and high-grade with signet ring cell tumours. From the epidemiology viewpoint, no clear differences in terms of age, gender, race or risk factors are reported. Although prognosis is generally much better than that characterising patients with peritoneal carcinomatosis (from tumours such as gastric, colorectal, pancreatic and ovarian carcinomas), survival rates can be different based upon histological grade (i.e. worse prognosis for patients with higher grade PMP) (De Vita 2008; Hinson 1998; Mukherjee 2004; Panarelli 2011; Ronnett 1997; Sherer 2001; Smeenk 2008; Smeenk 2008a; Young 2004).

\section{Description of the intervention}

The standard therapy for PMP is cytoreductive surgery (Gough 1994; Guner 2005; Mann 1990; Miner 2005; Moran 2008; Rubino 2012; Winder 2010). All tumour deposits onto the peritoneal surface are excised through the abdominal cavity together with segmental peritonectomy of the anatomical area affected by the PMP which include left diaphragm, right diaphragm, pelvis, left and right antero-lateral abdominal wall. Multivisceral resections may be needed during surgery, such as performance of radical omentectomy (i.e., complete removal of the greater omentum, the fatty apron-like peritoneal layer that covers the bowel loops), hystero-oophorectomy (i.e., removal of uterus and ovaries), and intestinal resections. The main goal of surgical resection is to relieve symptoms secondary to the tumour bulk and ascites (abnormal buildup of fluid in the abdomen) as well as to resect all tumour deposits in order to prolong patient survival. The degree of tumour debulking is the most important prognostic factor for survival in these patients (Gough 1994; Guner 2005; Miner 2005). In other words, a successful tumour resection, with no macroscopic disease left in the abdomen, is associated with the best prognosis. As mentioned above, PMP has a characteristic route of spreading through the abdomen, requiring accurate abdominal exploration and resections. Clearly, preoperative evaluation through contrastenhanced computerised tomography (CT) scan (and, in selected cases with meaningful pelvic involvement, magnetic resonance imaging (MRI) scan) can be of great help when planning a resection (Liu 2009).

Sugarbaker established a method, the peritoneal cancer index (PCI), which is the most widely used staging approach to PMP and peritoneal carcinomatosis from other neoplasms (Sugarbaker 1999; Sugarbaker 1993). The abdomen is classified using 12 regions, and a score from 0 (no tumour deposits) to 3 (tumour deposits of more than $5 \mathrm{~cm}$ or confluent) is assigned to each region. The PCI is calculated as the sum of each region score and offers important prognostic information, as higher scores are associated with greater probability of disease recurrence and survival (Sugarbaker 1996; Sugarbaker 1996a; Sugarbaker 2001).

The main criterion to preclude surgical resection is the presence of tumour deposits in the area of the hepato-duodenal ligament, a situation that needs to be ruled out with preoperative imaging and abdominal exploration (De Vita 2008). Local recurrence often occurs, which requires further surgical resection (Glehen 2004). In this regard it is important to minimise small and large bowel segmental resection in order to avoid long-term morbidity.

Despite thorough surgical resection, microscopic disease cannot be eradicated with surgery and intraperitoneal chemotherapy approaches have been established to reduce the amount of residual disease that cannot be identified both preoperatively and intraoperatively.

Hyperthermic intraperitoneal chemotherapy (HIPEC) has been proposed as an additional treatment to enhance local disease control achieved with cytoreductive surgery (Deraco 2003; Deraco 2004; Deraco 2007; Pilati 2001; Pilati 2003; Sugarbaker 2006; 
Sugarbaker 2009; Witkamp 2001). After optimal surgical cytoreduction, HIPEC is performed intra-operatively through insertion of catheters through the abdominal wall and used to administer cytotoxic drugs (i.e. chemotherapy) under hyperthermic conditions ranging between 40 and 41 degrees Celsius by means of an extracorporeal circuit (Brucher 2012; Bryant 2005). The most commonly used chemotherapy agents for HIPEC are cisplatin, mitomycin-C and 5-fluorouracil (Farquharson 2008; Jacquet 1998): these drugs are infused through the abdominal cavity - usually for 60 minutes - with the aim of targeting the microscopic PMP deposits that remain after cytoreductive surgery, thus reducing the risk of locoregional disease recurrence. The intraperitoneal drug concentrations reached during HIPEC are much higher than those reached by means of systemic chemotherapy, which is the main rational for every locoregional treatment (Brucher 2012; Sugarbaker 2006).

\section{How the intervention might work}

Cytoreductive surgery is aimed at resection of all visible tumour deposits. HIPEC allows cytotoxic drugs to reach concentrations in the peritoneal cavity that are approximately 20 times higher than those reached with systemic (i.e. intravenous) drug administration, without resulting in life-threatening toxicity. By targeting minimal residual disease not removed by surgery, HIPEC aims to virtually eradicate microscopic tumour deposits which may be left after surgery, with the ultimate goal of prolonging patient survival.

\section{Why it is important to do this review}

The therapeutic effectiveness of combining cytoreductive surgery with HIPEC is supported by randomised controlled trials for patients with ovarian cancer (Spiliotis 2015), colorectal cancer (Verwaal 2003), and gastric cancer (Yang 2011), but has never been formally proven in PMP. Nevertheless, optimal cytoreduction plus HIPEC is often considered to provide patients with the best therapeutic chance to control the PMP (Bryant 2004; Deraco 2004; Loungnarath 2005; Witkamp 2001a). Cytoreductive surgery and HIPEC can cause significant postoperative morbidity and mortality; in fact, candidates to these treatments must be counselled about the high morbidity risk. Therefore, when choosing this approach for the treatment of PMP, physicians should take into account not only the unproven synergy between cytoreduction and HIPEC but also the side effects of this combination. Finally, this procedure carries the risk of potential impairment of quality of life after surgery, due to the relatively high incidence of adhesion syndrome which may require reoperation (Kerscher 2010).

Although these issues have been addressed in qualitative reviews and expert opinion articles, a systematic approach has not yet been applied to this field of oncology, which has prompted us to fill this gap in the medical literature.

\section{O B J E C T I VES}

The aim of this work is to evaluate the efficacy of cytoreductive surgery alone versus cytoreductive surgery in combination with hyperthermic intraperitoneal chemotherapy (HIPEC), on patient benefits, complications and short-term outcomes in patients with pseudomyxoma peritonei (PMP).

\section{METHODS}

\section{Criteria for considering studies for this review}

\section{Types of studies}

We will include randomised controlled trials (RCTs), comparative non-randomised studies, controlled before-and-after studies (CBAs) and interrupted time series (ITS). No cluster-RCTs or cross-over RCTs will be considered. We will take into consideration potential confounders, such as type of surgery (only optimal cytoreduction or any cytoreduction) and origin of pseudomyxoma peritonei (appendix versus ovarian versus unknown/undefined/ unreported origin). No language restriction will be applied.

\section{Types of participants}

We will include adult participants (people aged 18 years or more, any sex) with a histological diagnosis of pseudomyxoma peritonei (PMP) who underwent cytoreductive surgery with or without hyperthermic intraperitoneal chemotherapy (HIPEC).

\section{Types of interventions}

Cytoreductive surgery alone will be considered the reference treatment, whereas cytoreductive surgery plus HIPEC will be considered the experimental treatment.

\section{Types of outcome measures}

\section{Primary outcomes}

- Overall survival: median survival and five-year survival rate. We will use the hazard ratio (HR) if a comparison between reference and experimental treatments is available.

- Cancer-specific survival: median survival and five-year survival rate. We will use the HR if a comparison between reference and experimental treatments is available.

- Postoperative morbidity and mortality: rates of morbidity (overall and severe, as defined by need for reoperation) and mortality within 30 days from treatment. We will use the odds 
ratio (OR) if a comparison between reference and experimental treatments is available; since HIPEC can be associated with prolonged postoperative ileus, the average length of hospital stay (in days) will be recorded for both cytoreductive surgery alone and combined with HIPEC,.

\section{Secondary outcomes}

- Disease-free survival: median survival and five-year survival rate.

- Quality of life: since there are no standardised diseasespecific scales and questionnaires to assess the quality of life of these patients undergoing HIPEC, we will describe findings from studies, if any.

\section{Search methods for identification of studies}

\section{Electronic searches}

We will search the Cochrane Central Register of Controlled trials (CENTRAL) (Appendix 1). Furthermore, we will search the electronic databases MEDLINE (Appendix 2) and Embase (Appendix 3). Further information will be searched for in clinical trial registers (ClinicalTrials.gov and UK Clinical Trials Gateway) as well as in review articles dedicated to this subject and references listed in each eligible article. There will be no restriction on language. Moreover, we will apply no time restrictions.

We will not consider unpublished data or findings reported in abstract form only, due to the lack of data that can be retrieved from these sources as opposed to the need to have as much information as possible in an ill-defined subject such as PMP.

In addition to the electronic search, we will contact authors of included studies for additional or missing information, as well as cross checking references of all included studies.

Two authors (SP and SM) will independently screen retrieved citations. Abstracts will be independently evaluated by the same two authors: any disagreement will be solved by discussion and consensus with a third author (AS). Based on abstract reading, two authors (SP and SM) will retrieve the full text of potentially eligible articles which will be screened for inclusion. Also in this case, any disagreement will be solved by discussion and consensus with a third author (AS).

\section{Data collection and analysis}

\section{Selection of studies}

We will assess the titles and abstracts of retrieved articles for eligibility. If this information is insufficient to assess eligibility, we will review the full article. Two review authors will be in charge of this task; the authors will achieve consensus on all results considered for the final analysis through iteration and discussion. Once all eligible studies are identified, the two authors will independently perform a quality evaluation of the heterogeneity between studies, randomisation (if applicable), blinding and follow-up.

\section{Data extraction and management}

Two review authors (SM and SP) will independently assess the included studies in accordance with the Cochrane Handbook for Systematic Reviews of Interventions (Higgins 2011).

The review authors will compare their evaluations and resolve possible inconsistencies. We will use a pilot data extraction form (including the characteristics of included studies, comprising information which allows for 'Risk of bias' judgements) for the first third of retrieved articles; we will then refine the form (if necessary) and use the refined version for the final data extraction from all retrieved articles.

\section{Assessment of risk of bias in included studies}

We will assess the risk of bias in included studies according to the recommendations in theCochrane Handbook (Higgins 2011), considering the following for RCTs:

- the method of generation of the randomisation sequence;

- the method of allocation concealment;

- the blinding of participants and personnel;

- the blinding of outcome assessors;

- the presence of incomplete outcome data;

- selective outcome reporting;

- other bias.

This information will be recorded in a 'Risk of bias' table, which is part of the Characteristics of included studies table for each study. We will report the overall risk of bias for each study as follows:

- low risk of bias (plausible bias unlikely to seriously alter the results) if all criteria mentioned above are assessed as being at low risk of bias;

- unclear risk of bias (plausible bias that raises some doubt about the results) if one or more criteria are assessed as being at unclear risk of bias; or

- high risk of bias (plausible bias that seriously weakens confidence in the results) if one or more criteria are assessed as being at high risk of bias.

As regards comparative but non-randomised studies, risk of bias will be evaluated by means of the ROBINS-I tool (Risk Of Bias In Non-randomised Studies - of Interventions) (Sterne 2016), which considers the following bias domains.

- Pre-intervention: 1) bias due to confounding; 2) bias in selection of participants into the study

- At intervention: 1) bias in classification of interventions

Cytoreductive surgery alone or combined with hyperthermic intraperitoneal chemotherapy (HIPEC) for pseudomyxoma peritonei 
- Post-intervention: 1) bias due to deviations from intended interventions; 2) bias due to missing data; 3) bias in measurement of outcomes; 4) bias in selection of the reported result

We will present data on the risk of bias in included studies in an additional table, and we will consider the 'Risk of bias' assessment when we interpret the results of the review. We will not exclude studies on the grounds of risk of bias. Where it is appropriate to undertake a meta-analysis, we will include risk of bias in our GRADE assessment of study limitations.

\section{Measures of treatment effect}

For survival comparisons (should data be permissive), hazard ratios (HRs) will be either entered directly in Review Manager (if directly reported in the eligible article, usually as a result of a Cox regression survival analysis) or extrapolated from Kaplan-Meier plots using the methods described by Parmar (Parmar 1998). For secondary outcomes, we will use morbidity rates and morbidity odds ratios (ORs). We will use $95 \%$ confidence intervals (CIs) as a measure of estimated uncertainty for all outcome measures.

\section{Unit of analysis issues}

The unit of analysis will be the individual participant. No clusterRCTs or cross-over RCTs will be considered. Although we do not expect to find this kind of studies, in case of multiple-arm trials we will only consider the comparison between the two arms of interest, that is, cytoreductive surgery alone and surgery combined with HIPEC,

\section{Dealing with missing data}

In case of missing data we will try to contact the study authors to retrieve those data. In case no data can be retrieved, we will apply the best-worst case scenario approach to assess the impact of missing data on findings, as suggested in the Cochrane Handbook (Higgins 2011). In particular, for dichotomous outcomes we will use a sensitivity analysis based on considering the so called "bestcase" and "worst-case" scenario: in the former scenario all participants with missing outcomes in the experimental intervention group are considered to have had good outcomes, and all those with missing outcomes in the control intervention group are considered to have had poor outcomes; in the "worst-case" scenario, the opposite situation is considered. The difference in pooled results between the two scenarios provides an estimate of the impact of missing data on the final results.

\section{Assessment of heterogeneity}

We will assess the consistency of results (effect sizes) among studies using the $\mathrm{I}^{2}$ statistic (Higgins 2002). To be more conservative, we will consider that heterogeneity is statistically significant when the Cochrane Q-test P value is less than 0.1 (i.e. the alpha level of significance for this test will be set at 10\%). Following recommendations in the Cochrane Handbook (Higgins 2011), we will interpret $\mathrm{I}^{2}$ as follows: $0 \%$ to $40 \%$ might not be important, $30 \%$ to $60 \%$ may represent moderate heterogeneity, $50 \%$ to $90 \%$ may represent substantial heterogeneity, $75 \%$ to $100 \%$ considerable heterogeneity. Where substantial heterogeneity is detected (I ${ }^{2}>50 \%$ ), we will investigate possible explanations for this informally and will summarise the data using a random-effects model, as appropriate.

For non-randomised studies, we will collect the confounding factors (typically, the baseline characteristics of the treated population) considered by the authors of the included articles, and we will record whether these confounding factors were taken into account by the study design or analysis (e.g. inclusion of confounding factors as covariates of regression or survival analyses).

\section{Assessment of reporting biases}

We will assess potential publication bias for the primary outcome with the use of funnel plots if there are more than 10 included studies, as recommended in Chapter 10 in the Cochrane Handbook for Systematic Reviews of Interventions (Sterne 2011).

\section{Data synthesis}

Where the population, intervention and outcome are sufficiently similar to allow pooling of data in a meta-analysis, we will perform meta-analysis following the guidance in the Cochrane Handbook (Higgins 2011; Moher 2009).

We will apply meta-analysis calculations (Sutton 2000), to evaluate the effect of the treatment of interest on the clinical outcomes previously specified.

We will present results for both treatment efficacy and toxicity as risk ratios (RRs) and their 95\% CIs.

Due to expected clinical heterogeneity (e.g. disease burden, previous treatments, extent of cytoreduction or debulking), we will use a random-effects model for creating the pooled estimates of each outcome measure (in case of homogeneity, the random-effects model results coincide with those of a fixed-effect model) (DerSimonian 1986).

We will perform statistical analyses using Cochrane's review-writing software, Review Manager 5 (RevMan 2014).

If no RCTs are available, we will report findings descriptively (acknowledging that it is generally considered inappropriate to metaanalyse data from observational studies (Smith 1999)). In particular, in case of data from non-comparative studies, we will record survival data as three-year, five-year and 10-year survival rates; as regards adverse events, we will describe their rate during hospitalisation. We will group data by intervention type and type of participant, as appropriate. 


\section{GRADE assessment}

We will assess the quality of the evidence using the GRADE system (Guyatt 2011). Briefly, quality of evidence is graded into four levels: high, moderate, low, and very-low quality. Evidence from RCTs is generally considered high quality; however the quality can be downgraded by one level (serious concern) or two levels (very serious concern) for the following reasons: risk of bias, inconsistency (unexplained heterogeneity, inconsistency of results), indi- rectness (indirect population, intervention, control, outcomes), and imprecision (wide confidence intervals, confidence intervals crossing the null value: that is, the results are compatible with both favourable and dangerous effects).

Evidence from non-randomised studies is generally graded as low or very low quality, but it can be upgraded if the aggregated data show large effects or a dose response relationship (Higgins 2011). Overall, we will apply the following rules (as per Cochrane Handbook guidelines):

Randomised controlled trials; or double-upgraded observational High studies

Downgraded randomised controlled trials; or upgraded observa- Moderate tional studies

Double-downgraded randomised controlled trials; or observa- Low tional studies

Triple-downgraded randomised controlled trials; or downgraded Very low observational studies; or case series/case reports

Should meta-analysis be feasible, we will include a 'Summary of findings' table (including number needed to treat, calculated as 1/ absolute risk reduction) which will report on both primary and secondary outcomes. The baseline risk will be the average event rate in the non-treatment (placebo or observation) arms of the included trials. Should meta-analysis be unfeasible, we will create a narrative 'Summary of findings' table.

\section{Subgroup analysis and investigation of heterogeneity}

We will use subgroup analysis and meta-regression to reveal potential sources of heterogeneity. Predefined subgroups will be defined by geographical origin of the trial (ethnicity), origin of tumour, line of treatment (first versus subsequent), sample size, tumour grade, tumour burden (possibly using the peritoneal cancer index proposed by Sugarbaker 1999, as mentioned in Description of the intervention), extent of cytoreduction, and chemotherapy used during HIPEC. In order to assess the effect of sample size, we will conduct meta-regression using sample size as the covariate of interest. Moreover, we will analyse adjusted and unadjusted data separately and compare them to each other in order to investigate the potential role of confounders.

\section{Sensitivity analysis}

We will use sensitivity analyses (e.g. leave-one-out procedure, which explores the extent to which the combined risk estimate might be affected by individual studies, assessed by consecutively omitting every study from the meta-analysis) to reveal potential sources of heterogeneity. To this aim, low-quality studies, studies only reporting unadjusted data, and studies with unclear definition of PMP will be excluded from analysis to investigate their impact on the summary statistics. Should we find more than 10 eligible trials, we will also assess publication bias by means of a funnel plot.

\section{ACKNOWLEDGEMENTS}

We would like to thank Henning Keinke Andersen, Sys Johnsen, Cochrane Colorectal Cancer editorial board, statisticians, and Fiona Chionh for peer review, for their help and advice for composing this protocol. 


\section{REFERE N C E S}

\section{Additional references}

\section{Brucher 2012}

Brücher BL, Piso P, Verwaal V, Esquivel J, Derraco M, Yonemura Y, et al. Peritoneal carcinomatosis: cytoreductive surgery and HIPEC-overview and basics. Cancer Investigation 2012;30:209-24.

\section{Bryant 2004}

Bryant J, Clegg AJ, Sidhu MK, Brodin H, Royle P, Davidson P. Clinical effectiveness and costs of the Sugarbaker procedure for the treatment of pseudomyxoma peritonei. Health Technology Assessment 2004;8(7):iii, 1-54.

Bryant 2005

Bryant J, Clegg AJ, Sidhu MK, Brodin H, Royle P, Davidson P. Systematic review of the Sugarbaker procedure for pseudomyxoma peritonei. The British Journal of Surgery 2005;92(2):153-8.

Carr 2012

Carr NJ, Finch J, Ilesley IC, Chandrakumaran K, Mohamed F, Mirnezami A, et al. Pathology and prognosis in pseudomyxoma peritonei: a review of 274 cases. Journal of Clinical Pathology 2012;65:919-23.

Carr 2016

Carr NJ, Cecil TD, Mohamed F, Sobin LH, Sugarbaker PH, Gonzalez-Moreno S, et al. A Consensus for Classification and Pathologic Reporting of Pseudomyxoma Peritonei and Associated Appendiceal Neoplasia: The Results of the Peritoneal Surface Oncology Group International (PSOGI) Modified Delphi Process. The American journal of surgical pathology 2016;40(1):14-26. [PUBMED: 26492181]

\section{De Vita 2008}

Pingpank JF. Diagnosis and treatment of peritoneal carcinomatosis. In: De Vita TA, Lawrence, TS, Rosenberg SA editor(s). Cancer. Principles \& Practice of Oncology. 8th Edition. Vol. 2, Lippincott Williams \& Wilkins, 2008: 2389-99.

\section{Deraco 2003}

Deraco M, De Simone M, Rossi CR, Cavaliere F, Di Filippo F, Vaira M, et al. An Italian Multicentric Phase II study on peritonectomy and intra peritoneal hyperthermic perfusion (IPHP) to treat patients with pseudomyxoma peritonei. Journal of experimental \& clinical cancer research: CR 2003; 22(4 Suppl):35-9. [PUBMED: 16767904]

Deraco 2004

Deraco M, Baratti D, Inglese MG, Allaria B, Andreola $S$, Gavazzi C, et al. Peritonectomy and intraperitoneal hyperthermic perfusion (IPHP): a strategy that has confirmed its efficacy in patients with pseudomyxoma peritonei. Annals of Surgical Oncology 2004;11(4):393-8.

Deraco 2007

Deraco M, Baratti D, Kusamura S. Morbidity and quality of life following cytoreduction and HIPEC. Cancer Treatment and Research 2007;134:403-18.
DerSimonian 1986

DerSimonian R, Laird N. Meta-analysis in clinical trials. Controlled Clinical Trials 1986;7(3):177-88.

\section{DeSantis 2017}

DeSantis CE, Kramer JL, Jemal A. The burden of rare cancers in the United States. CA: a cancer journal for clinicians 2017;67(4):261-72. [PUBMED: 28542893]

\section{Farquharson 2008}

Farquharson AL, Pranesh N, Witham G, Swindell R, Taylor MB, Renehan AG, et al. A phase II study evaluating the use of concurrent mitomycin $\mathrm{C}$ and capecitabine in patients with advanced unresectable pseudomyxoma peritonei. British Journal of Cancer 2008;99:591-6.

\section{Glehen 2004}

Glehen O, Mohamed F, Gilly FN. Peritoneal carcinomatosis from digestive tract cancer: new management by cytoreductive surgery and intraperitoneal chemohyperthermia. Lancet Oncology 2004;5(4):219-28.

Gough 1994

Gough DB, Donohue JH, Schutt AJ, Gonchoroff N, Goellner JR, Wilson TO, et al. Pseudomyxoma peritonei. Long-term patient survival with an aggressive regional approach. Annals of Surgery 1994;219(2):112-9.

\section{Guner 2005}

Guner Z, Schmidt U, Dahlke MH, Schlitt HJ, Klempnauer J, Piso P. Cytoreductive surgery and intraperitoneal chemotherapy for pseudomyxoma peritonei. International Journal of Colorectal Disease 2005;20(2):155-60. Epub 2004 Oct 16.

Guyatt 2011

Guyatt GH, Oxman AD, Schunemann HJ, Tugwell P, Knottnerus A. GRADE guidelines: a new series of articles in the Journal of Clinical Epidemiology. Journal of Clinical Epidemiology 2011;64:380-2.

\section{Higgins 2002}

Higgins JP, Thompson SG. Quantifying heterogeneity in a meta-analysis. Statistics in Medicine 2002;21:1539-58.

\section{Higgins 2011}

Higgins JPT, Green S (editors). Cochrane Handbook for Systematic Reviews of Interventions Version 5.1.0 (updated March 2011). The Cochrane Collaboration, 2011. Available from www.cochrane-handbook.org

\section{Hinson 1998}

Hinson FL, Ambrose NS. Pseudomyxoma peritonei. The British Journal of Surgery 1998;85(10):1332-9.

\section{Jacquet 1998}

Jacquet P, Averbach A, Stephens AD, Stuart OA, Chang $\mathrm{D}$, Sugarbaker $\mathrm{PH}$. Heated intraoperative intraperitoneal mitomycin $\mathrm{C}$ and early postoperative intraperitoneal 5fluorouracil: pharmacokinetic studies. Oncology 1998;55 (2):130-8.

Cytoreductive surgery alone or combined with hyperthermic intraperitoneal chemotherapy (HIPEC) for pseudomyxoma peritone 


\section{Kerscher 2010}

Kerscher AG, Mallalieu J, Pitroff A, Kerscher F, Esquivel J. Morbidity and mortality of 109 consecutive cytoreductive procedures with hyperthermic intraperitoneal chemotherapy (HIPEC) performed at a community hospital. World Journal of Surgery 2010;34:62-9.

Liu 2009

Liu H, Buckner RL, Talukdar T, Tanaka N, Madsen JR, Stufflebeam SM. Task-free presurgical mapping using functional magnetic resonance imaging intrinsic activity. Journal of Neurosurgery 2009;111(4):746-54.

\section{Loungnarath 2005}

Loungnarath R, Causeret S, Bossard N, Faheez M, SayagBeaujard AC, Brigand C, et al. Cytoreductive Surgery With Intraperitoneal Chemohyperthermia for the Treatment of Pseudomyxoma Peritonei: A Prospective Study. Diseases of Colon \& Rectum 2005;10:10.

\section{Mann 1990}

Mann WJ Jr, Wagner J, Chumas J, Chalas E. The management of pseudomyxoma peritonei. Cancer 1990;66 (7):1636-40.

\section{McKenney 2008}

McKenney JK, Soslow RA, Longacre TA. Ovarian mature teratomas with mucinous epithelial neoplasms: morphologic heterogeneity and association with pseudomyxoma peritonei. The American journal of surgical pathology 2008; 32(5):645-55. [PUBMED: 18344868]

\section{Miner 2005}

Miner TJ, Shia J, Jaques DP, Klimstra DS, Brennan MF, Coit DG. Long-term survival following treatment of pseudomyxoma peritonei: an analysis of surgical therapy. Annals of Surgery 2005;241(2):300-8.

\section{Moher 2009}

Moher D, Liberati A, Tetzlaff J, Altman DG. Preferred Reporting Items for Systematic Reviews and Meta-Analyses: The PRISMA Statement. PLoS Medicine 2009;6:e1000097.

\section{Moran 2003}

Moran BJ, Cecil TD. The etiology, clinical presentation, and management of pseudomyxoma peritonei. Surgical Oncology Clinics of North American 2003;12(3):585-603

\section{Moran 2008}

Moran B, Baratti D, Yan TD, Kusamura S, Deraco $\mathrm{M}$. Consensus statement on the loco-regional treatment of appendiceal mucinous neoplasms with peritoneal dissemination (pseudomyxoma peritonei). Journal of Surgical Oncology 2008;98:277-82.

\section{Mukherjee 2004}

Mukherjee A, Parvaiz A, Cecil TD, Moran BJ. Pseudomyxoma peritonei usually originates from the appendix: a review of the evidence. European Journal of Gynaecological Oncology 2004;25(4):411-4.

\section{Panarelli 2011}

Panarelli NC, Yantiss RK. Mucinous neoplasms of the appendix and peritoneum. Archives of Pathology and Laboratory Medicine 2011;135:1261-8.

\section{Parmar 1998}

Parmar MK, Torri V, Stewart L. Extracting summary statistics to perform meta-analyses of the published literature for survival endpoints. Statistics in Medicine 1998; 17:2815-34.

\section{Pilati 2001}

Pilati P, Rossi CR, Mocellin S, Foletto M, Scagnet B, Pasetto $\mathrm{L}$, et al. Multimodal treatment of peritoneal carcinomatosis and sarcomatosis. European Journal of Surgical Oncology 2001;27:125-34.

\section{Pilati 2003}

Pilati P, Mocellin S, Rossi CR, Foletto M, Campana L, Nitti D, et. al. Cytoreductive surgery combined with hyperthermic intraperitoneal intraoperative chemotherapy for peritoneal carcinomatosis arising from colon adenocarcinoma. Annals of Surgical Oncology 2003;10: $508-13$

\section{RevMan 2014 [Computer program]}

The Nordic Cochrane Centre, The Cochrane Collaboration. Review Manager (RevMan) [Computer program]. Version 5.3. Copenhagen:. The Nordic Cochrane Centre, The Cochrane Collaboration, 2014.

\section{Ronnett 1997}

Ronnett BM, Shmookler BM, Sugarbaker PH, Kurman RJ. Pseudomyxoma peritonei: new concepts in diagnosis, origin, nomenclature, and relationship to mucinous borderline (low malignant potential) tumors of the ovary. Anatomic Pathology 1997;2:197-226.

\section{Ronnett 2001}

Ronnett BM, Yan H, Kurman RJ, Shmookler BM, Wu L, Sugarbaker PH. Patients with pseudomyxoma peritonei associated with disseminated peritoneal adenomucinosis have a significantly more favorable prognosis than patients with peritoneal mucinous carcinomatosis. Cancer 2001;92 (1):85-91.

\section{Rubino 2012}

Rubino MS, Abdel-Misih RZ, Bennett JJ, Petrelli NJ. Peritoneal surface malignancies and regional treatment: a review of the literature. Surgical Oncology 2012;21:87-94.

\section{Sherer 2001}

Sherer DM, Abulafia O, Eliakim R. Pseudomyxoma peritonei: a review of current literature. Gynecological and Obstetric Investigation 2001;51(2):73-80.

\section{Smeenk 2008}

Smeenk RM, van Velthuysen ML, Verwaal VJ, Zoetmulder FA. Appendiceal neoplasms and pseudomyxoma peritonei: a population based study. European Journal of Surgical Oncology 2008;34:196-201.

\section{Smeenk 2008a}

Smeenk RM, Bruin SC, van Velthuysen ML, Verwaal VJ. Pseudomyxoma peritonei. Current Problems in Surgery 2008;45:527-75.

\section{Smith 1992}

Smith JW, Kemeny N, Caldwell C, Banner P, Sigurdson E, Huvos A. Pseudomyxoma peritonei of appendiceal origin. 
The Memorial Sloan-Kettering Cancer Center experience. Cancer 1992;70(2):396-401.

\section{Smith 1999}

Smith GD, Egger M. Meta-analyses of observational data should be done with due care. BMJ 1999;318(7175):56.

\section{Spiliotis 2015}

Spiliotis J, Halkia E, Lianos E, Kalantzi N, Grivas A, Efstathiou E, et. al. Cytoreductive surgery and HIPEC in recurrent epithelial ovarian cancer: a prospective randomized phase III study. Annals of Surgical Oncology 2015;22:1570-5.

\section{Sterne 2011}

Sterne JAC, Egger M, Moher D (editors). Chapter 10: Addressing reporting biases. In: Higgins JPT, Green $S$ (editors). Cochrane Handbook for Systematic Reviews of Intervention. Version 5.1.0 (updated March 2011). Available from www.cochrane-handbook.org.

\section{Sterne 2016}

Sterne JA, Hernán MA, Reeves BC, Savovi

ć J, Berkman ND, Viswanathan M et. al. ROBINS-I: a tool for assessing risk of bias in non-randomised studies of interventions. BMJ 2016;355:14919.

\section{Stewart 2014}

Stewart CJ, Ardakani NM, Doherty DA, Young RH. An evaluation of the morphologic features of low-grade mucinous neoplasms of the appendix metastatic in the ovary, and comparison with primary ovarian mucinous tumors. International journal of gynecological pathology: official journal of the International Society of Gynecological Pathologists 2014;33(1):1-10. [PUBMED: 24300528]

Sugarbaker 1993

Sugarbaker PH, Zhu BW, Sese GB, Shmookler B. Peritoneal carcinomatosis from appendiceal cancer: results in 69 patients treated by cytoreductive surgery and intraperitoneal chemotherapy. Diseases of the Colon \& Rectum 1993;36(4): 323-9.

Sugarbaker 1994

Sugarbaker PH. Pseudomyxoma peritonei. A cancer whose biology is characterized by a redistribution phenomenon. Annals of Surgery 1994;219(2):109-11.

\section{Sugarbaker 1996}

Sugarbaker PH, Fernandez-Trigo V, Shamsa F. Clinical determinants of treatment failure in patients with pseudomyxoma peritonei. Cancer Treatment and Research 1996;81:121-32.

\section{Sugarbaker 1996a}

Sugarbaker PH, Ronnett BM, Archer A, Averbach AM, Bland R, Chang D, et al. Pseudomyxoma peritonei syndrome. Advances in Surgery 1996;30:233-80.

\section{Sugarbaker 1999}

Sugarbaker PH. Successful management of microscopic residual disease in large bowel cancer. Cancer chemotherapy and pharmacology 1999;43 Suppl:S15-25. [PUBMED: 10357554]

\section{Sugarbaker 2001}

Sugarbaker PH. Cytoreductive surgery and perioperative intraperitoneal chemotherapy as a curative approach to pseudomyxoma peritonei syndrome. Tumori 2001;87(4): S3-5.

Sugarbaker 2006

Sugarbaker PH. New standard of care for appendiceal epithelial neoplasms and pseudomyxoma peritonei syndrome?. The Lancet Oncology 2006;7:69-76.

\section{Sugarbaker 2009}

Sugarbaker PH. Comprehensive management of peritoneal surface malignancy using cytoreductive surgery and perioperative intraperitoneal chemotherapy: the Washington Cancer Institute approach. Expert Opinion on Pharmacotherapy 2009;10:1965-77.

Sutton 2000

Sutton A, Abrams K, Jones D, Sheldon T, Song. Methods for Meta-analysis in Medical Research. John Wiley \& Sons, 2000

Verwaal 2003

Verwaal VJ, van Ruth S, de Bree E, van Sloothen GW, van Tinteren $\mathrm{H}$, Boot $\mathrm{H}$, et al. Randomized trial of cytoreduction and hyperthermic intraperitoneal chemotherapy versus systemic chemotherapy and palliative surgery in patients with peritoneal carcinomatosis of colorectal cancer. Journal of Clinical Oncology 2003;21(20): $3737-43$.

\section{Winder 2010}

Winder T, Lenz HJ. Mucinous adenocarcinomas with intra-abdominal dissemination: a review of current therapy. The Oncologist 2010;15:836-44.

\section{Witkamp 2001}

Witkamp AJ, de Bree E, Van Goethem R, Zoetmulder FA. Rationale and techniques of intra-operative hyperthermic intraperitoneal chemotherapy. Cancer Treatment Reviews 2001;27(6):365-74.

\section{Witkamp 2001a}

Witkamp AJ, de Bree E, Kaag MM, van Slooten GW, van Coevorden F, Zoetmulder FA. Extensive surgical cytoreduction and intraoperative hyperthermic intraperitoneal chemotherapy in patients with pseudomyxoma peritonei. The British Journal of Surgery 2001;88(3):458-63.

Yang 2011

Yang XJ, Huang CQ, Suo T, Mei LJ, Yang GL, Cheng FL, et. al. Cytoreductive surgery and hyperthermic intraperitoneal chemotherapy improves survival of patients with peritoneal carcinomatosis from gastric cancer: final results of a phase III randomized clinical trial. Annals of Surgical Oncology 2011;18:1575-81.

\section{Young 2004}

Young RH. Pseudomyxoma peritonei and selected other aspects of the spread of appendiceal neoplasms. Seminars in Diagnostic Pathology 2004;21(2):134-50.

* Indicates the major publication for the study

Cytoreductive surgery alone or combined with hyperthermic intraperitoneal chemotherapy (HIPEC) for pseudomyxoma peritonei 


\section{A P P E N D I C E S}

\section{Appendix I. Cochrane Central Register of Controlled Trials (CENTRAL), search strategy}

\section{The Cochrane Library, CENTRAL}

\#1 MeSH descriptor: [Pseudomyxoma Peritonei] explode all trees

\#2 pseudomyx* periton*:ti,ab,kw

\#3 (peritoneal adenomucinosis or DPAM or peritoneal mucinous carcinomatosis or PMCA):ti,ab,kw

\#4 MeSH descriptor: [Cytoreduction Surgical Procedures] explode all trees

\#5 cytoreduct* surg* $^{*}$ ti,ab,kw

\#6 (intraperitoneal chemotherap* or heated intraperitoneal chemotherap* or hypertherm* intraperitoneal chemotherap* or HIPEC):

ti,ab, kw

$\# 7 \# 1$ or \#2 or \#3

$\# 8 \# 4$ or $\# 5$ or \#6

$\# 9 \# 7$ and \#8

\section{Appendix 2. MEDLINE (Ovid) 1950 to present, search strategy}

\section{MEDLINE (Ovid) 1950 to present}

1. Exp Pseudomyxoma peritonei /

2. pseudomyx* periton*.mp.

3. (peritoneal adenomucinosis or DPAM or peritoneal mucinous carcinomatosis or PMCA).mp.

4. 1 or 2 or 3

5. Exp Cytoreduction surgical procedures/

6. Cytoreduct* surg*.mp.

7. (intraperitoneal chemotherap* or heated intraperitoneal chemotherap* or hypertherm* intraperitoneal chemotherap* or HIPEC).mp.

8. 5 or 6 or 7

9.4 and 8

\section{Appendix 3. Embase (Ovid) 1974 to present, search strategy}

\section{Embase (Ovid) 1974 to present}

1. Exp peritoneum pseudomyxoma/

2. Pseudomyx* periton*.mp.

3. (peritoneal adenomucinosis or DPAM or peritoneal mucinous carcinomatosis or PMCA).mp.

4. 1 or 2 or 3

5. Exp cytoreductive surgery/

6. Cytoreduct* surg*.mp.

7. (intraperitoneal chemotherap* or heated intraperitoneal chemotherap* or hypertherm* intraperitoneal chemotherap* or HIPEC).mp.

8. 5 or 6 or 7

9. 4 and 8 
WHAT'S NEW

\begin{tabular}{l|l|l}
\hline Date & Event & Description \\
\hline 3 September 2018 & Amended & Byline details changed \\
\hline 13 August 2018 & New citation required and major changes & Amended and copy edited version, complying to MECIR \\
\hline
\end{tabular}

\section{CONTRIBUTIONS OFAUTHORS}

All authors have contributed to the final draft of this updated protocol.

\section{DECLARATIONSOF INTEREST}

Sandro Pasquali (SP): none known

Antonio Sommariva (AS): none known

Haile Mahteme (HM): none known

Tao Suo (TS): none known

Huaixing $\mathrm{Ma}(\mathrm{HM})$ : none known

Saveria Tropea (ST): none known

Josephine Lyngh Steenberg (JLS): none known

Simone Mocellin (SM): none known 